\title{
How do plants make mitochondria?
}

\author{
Chris Carrie • Monika W. Murcha • Estelle Giraud • \\ Sophia Ng $\cdot$ Ming Fang Zhang $\cdot$ Reena Narsai $\cdot$ \\ James Whelan
}

Received: 2 August 2012 / Accepted: 4 September 2012 / Published online: 14 September 2012

(C) Springer-Verlag 2012

\begin{abstract}
Plant mitochondria can differ in size, shape, number and protein content across different tissue types and over development. These differences are a result of signaling and regulatory processes that ensure mitochondrial function is tuned in a cell-specific manner to support proper plant growth and development. In the last decade, the processes involved in mitochondrial biogenesis are becoming clearer, including; how dormant seeds transition from empty promitochondria to fully functional mitochondria with extensive cristae structures and various biochemical
\end{abstract}

A contribution to the Special Issue on Evolution and Biogenesis of Chloroplasts and Mitochondria.

C. Carrie $(\square)$

Department of Biology I, Botany, Ludwig-Maximilians

Universität München, Großhaderner Strasse 2-4,

82152 Planegg-Martinsried, Germany

e-mail: christopher.carrie21@gmail.com

M. W. Murcha $\cdot$ E. Giraud · S. Ng · R. Narsai $\cdot$ J. Whelan ARC Centre of Excellence in Plant Energy Biology, Bayliss Building M316, University of Western Australia, 35 Stirling Highway, Crawley, WA 6009, Australia

S. Ng

Joint Research Laboratory in Genomics and Nutriomics, College of Life Sciences, Zhejiang University,

Hangzhou 310058, People's Republic of China

\section{F. Zhang}

Laboratory of Genetic Resources and Functional Improvement for Horticultural Plants, Department of Horticulture, Zhejiang University, Hangzhou 310029, People's Republic of China

\section{R. Narsai}

Centre for Computational Systems Biology, Bayliss Building M316, University of Western Australia, 35 Stirling Highway, Crawley, WA 6009, Australia activities, the regulation of nuclear genes encoding mitochondrial proteins via regulators of the diurnal cycle in plants, the mitochondrial stress response, the targeting of proteins to mitochondria and other organelles and connections between the respiratory chain and protein import complexes. All these findings indicate that mitochondrial function is a part of an integrated cellular network, and communication between mitochondria and other cellular processes extends beyond the known exchange or transport of metabolites. Our current knowledge now needs to be used to gain more insight into the molecular components at various levels of this hierarchical and complex regulatory and communication network, so that mitochondrial function can be predicted and modified in a rational manner.

Keywords Dual targeting $\cdot$ Mitochondria $\cdot$ Mitochondrial biogenesis · Post-transcriptional regulation · Protein import $\cdot$ Transcriptional regulation
Abbreviations
ABF4
Abscisic acid responsive elements binding factor 4
ABI4 Abscisic acid insensitive 4
$\mathrm{AP} 2 / \mathrm{ERF}$ Ethylene responsive element binding protein
bZIP Basic leucine zipper
CAREs Cis-acting regulatory elements
DGS-1 Digalactosyl diacylglycerol deficient suppressor 1
DRPs Dynamin-related proteins
MPP Mitochondrial processing peptidase
NCA2 Nuclear control of ATPase 2
Ndufs4 NADH dehydrogenase ubiquinone fragment 4
PMD Peroxisomal and mitochondrial division factor
PRAT Preprotein and amino acid transporter
PRR Pseudo response regulator 1
Rpotmp RNA polymerase of the mitochondria and plastid
TIM Translocase of the inner membrane 
Tim17 Translocase of the inner membrane protein of $17 \mathrm{kDa}$

Tim21 Translocase of the inner membrane protein of $21 \mathrm{kDa}$

\section{Introduction}

Mitochondria are present in virtually all eukaryotic cells and have essential roles in energy metabolism, various metabolic pathways and cell death. A legacy of the endosymbiotic origin of mitochondria is that they contain their own genome that encodes about 50 proteins in plants, and a variety of rRNA and tRNAs (Millar et al. 2011). The remainder and majority of the greater than 1,000 proteins located in the mitochondrion are encoded by nuclear genes, which are imported into mitochondria after translation in the cytosol. In all cases, the proteins encoded in the mitochondrion are found in multi-subunit complexes, also made up of nuclearencoded subunits. Mitochondria are not uniform or static entities within the cell; they can vary in number, composition and function between different cells, organs, and species over development and in response to different stresses.

In plants, many differences in mitochondrial numbers and characteristics have been observed and described. For example, proteomic analyses of isolated mitochondria from different organ/tissue types demonstrated that the mitochondrial proteome varies greatly between different organs/ tissue types (Lee et al. 2008, 2012). Analyses of the major oxidative phosphorylation complexes in mitochondria from different tissues demonstrated that the protein abundance of complex I is relatively high in leaves and stems but much lower in roots and callus tissue (Peters et al. 2012). In contrast, complex II abundance was found to be the inverse, as it was high in roots and callus tissue and lower in abundance in leaves and stems (Peters et al. 2012). In Arabidopsis, the number of mitochondria per cell was found to increase with cell size and over development (Preuten et al. 2010). Young leaves were observed to contain approximately 300 mitochondria per cell while older mature leaves were observed to have 450 mitochondria per cell (Preuten et al. 2010). Variations in mitochondrial morphology and dynamics are also evident, with the movement, fusion and fission of mitochondria documented (Logan 2010).

Overall, the term mitochondrial number is probably not the best way to describe the mitochondrial content of a cell, as it changes. Rather, mitochondria in a cell can be viewed as a dynamic population of parts to form the chondriome of the cell (Logan 2010). The questions remain: what triggers or signals an increase or decrease in mitochondrial mass in a cell, how are these signals transmitted and mechanistically acted upon in a cell (Fig. 1)? While the answers to these questions are not known, studies from a variety of sources have revealed insights into these processes in the last decade, which serve as a good foundation for tackling some outstanding fundamental questions concerning mitochondrial biogenesis in plants in the future.

\section{Mitochondrial biogenesis during germination}

Two models can be envisaged to account for mitochondrial biogenesis in cells. One model is the growth and division model, which describes how mitochondria change under different cellular conditions (Nisoli et al. 2004). Within the growth and division model, existing mitochondria are adapted to the demands of cell by incorporating more or different proteins (Nisoli et al. 2004). The second model is the maturation model, which proposes that a cell has a population of promitochondria, (i.e. stem mitochondria by analogy to stem cells), which exist in an undifferentiated state and can be differentiated into mature organelles in response to cellular signals (Plattner et al. 1970). Initial studies on mitochondrial biogenesis were carried out in wheat, where the distance from the basal meristem represents a development gradient. These studies revealed that mitochondrial proteins were made early in development (Ehrenshaft and Brambl 1990; Topping and Leaver 1990). Using a similar model in barley, more detailed studies confirmed that all mitochondrial proteins increased in abundance from the basal meristem to the end of the elongation zone, and afterwards could be divided into sub-groups, with some proteins then decreasing in abundance, while others, notably the subunits of glycine decarboxylase, increasing in abundance at the tip of the leaf (Thompson et al. 1998). The patterns observed could be readily explained by the physiology of the leaf, and the increase in the subunits of glycine decarboxylase was coincident with the increase in photosynthetic activity.

Seed germination provides an excellent model for the study of mitochondrial biogenesis, as mitochondria lack cristae in dry seeds, but upon imbibition develop into cristae containing mitochondria, in as little as $12 \mathrm{~h}$ (Logan et al. 2001; Howell et al. 2006, 2007). Thus, to some degree, there is synchrony in mitochondrial biogenesis in the earliest stages of germination. Studies in maize revealed that the transcripts encoding proteins in the ATP synthase complex and cytochrome oxidase accumulate after $6 \mathrm{~h}$ of imbibition (Ehrenshaft and Brambl 1990). Another study showed that transcripts for mitochondrial Hsp60 are present in dry seeds and increase upon imbibition (Prasad and Stewart 1992). Further studies using maize and rice show that the promitochondria develop into more typical mature mitochondria within 12-24 h after imbibition and this structural change is accompanied by an increase in metabolic activity (Logan et al. 2001; Howell et al. 2006, 2007). 
Fig. 1 What is known now and outstanding questions in regards to plant mitochondrial biogenesis? a It is known that a number of different transcription factors bind and regulate expression of genes encoding mitochondrial proteins. The mitochondrial proteome contains approximately 1,000 proteins; however, a number of these are also targeted to other organelles. It has been estimated that 20 proteins are dual targeted to mitochondria and peroxisomes and 100 proteins are dual targeted to mitochondria and chloroplasts. Interactions between mitochondrial import components and the respiratory chain can also affect mitochondrial biogenesis.

b What are the signals involved in the regulation of mitochondrial biogenesis, in terms of number, size, morphology, protein content and function? These signals may come from outside and act on the nucleus affecting nuclear gene expression (anterograde regulation). Do the signals originate from the organelles themselves, sending signals between organelles and back to the nucleus (retrograde regulation)? Is the dual targeting of proteins regulated in plant cells? There are all questions that remain unanswered
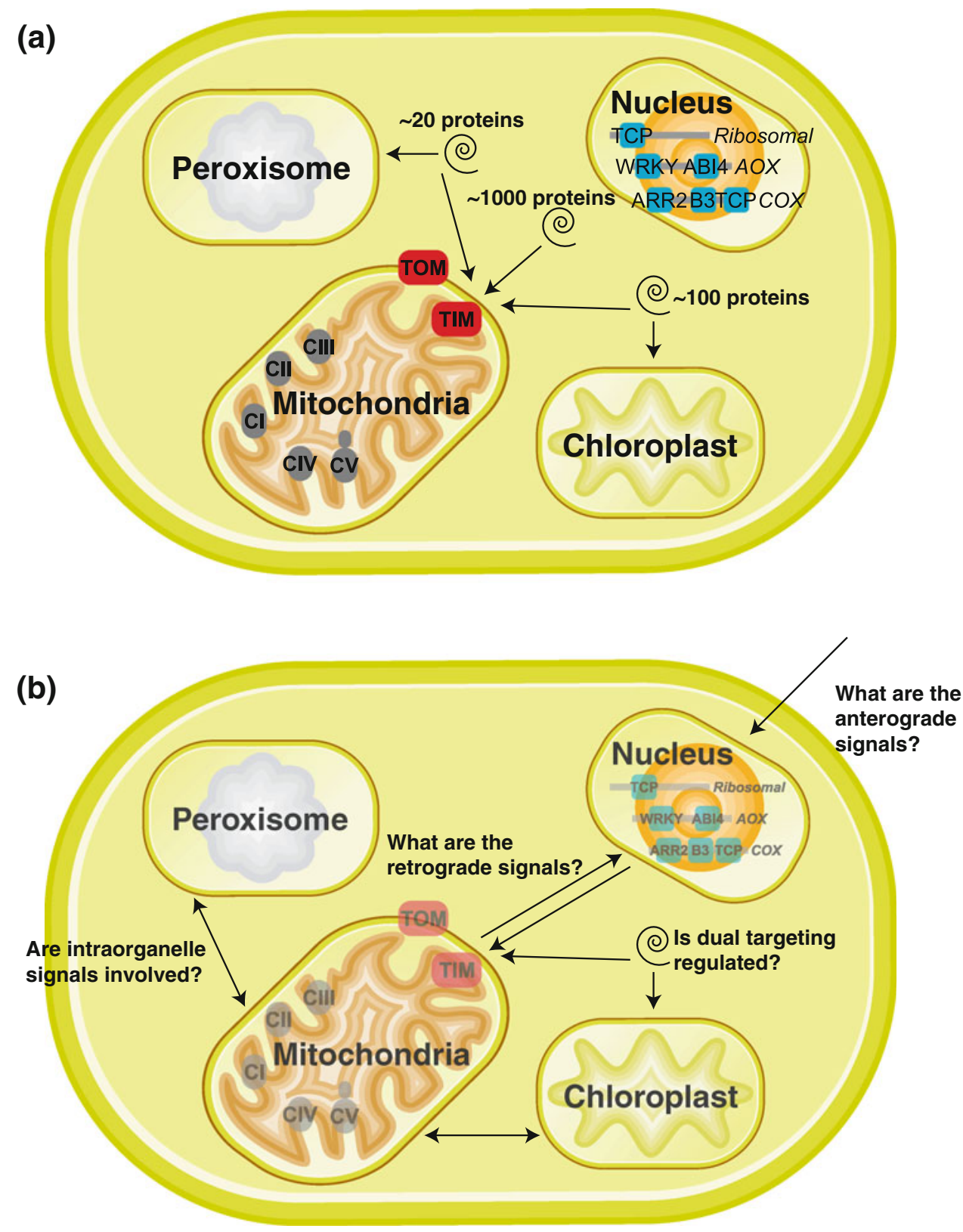

Studies in rice suggest an ordered assembly of mitochondrial components are required to build and/or import mitochondrial proteins present in promitochondria and are active almost immediately following imbibition, facilitating the rapid rate of mitochondrial biogenesis associated with the increases in respiration over the first $24 \mathrm{~h}$ of imbibition (Howell et al. 2006). It was observed that transcripts encoding proteins involved in the electron transport chain and TCA cycle components increased over time while transcripts encoding mitochondrial import apparatus components were highest in the earlier time points after imbibition (Howell et al. 2006). Along with changes in transcript levels, dynamic changes in mitochondrial protein content and function were also observed during rice germination
(Howell et al. 2006). Proteins of the mitochondrial import apparatus were found to be most abundant in dry seeds and decreased in abundance over time (Howell et al. 2006). In the latter stages of mitochondrial biogenesis, it was observed that the capacity of organic acid metabolism and appearance of TCA cycle components were increased, along with the coordination of the electron transport chain assembly components (Howell et al. 2006).

Using the resources available in Arabidopsis, recent studies have carried out detailed temporal transcriptome analysis of the transcriptome changes from freshly harvested dry seeds, through stratification (the moist chilling of seeds in darkness to break dormancy), through germination (the time from the start of water uptake to the point 
where the embryo emerges from the testa) and postgermination (Narsai et al. 2011; Law et al. 2012). This was combined with analysis of both mitochondrial and plastidencoded organellar gene expression (through qRT-PCR), data linking cellular development through changes in organelle size, number and morphology (through fluorescent tagging), and protein abundance measurements (through Western blotting and peptide mass spectrometry), giving the most in-depth view of mitochondrial biogenesis in germinating seeds (Narsai et al. 2011; Law et al. 2012).

It was observed that in Arabidopsis, mitochondria of typical size and oval morphology were evident after $12 \mathrm{~h}$ of imbibition under continuous light conditions (Law et al. 2012). The change from dormant promitochondria to metabolically active mature mitochondria was facilitated through a molecular switch, represented by a transient burst in the expression of genes encoding mitochondrial proteins (Narsai et al. 2011; Law et al. 2012). This burst of expression was over-represented in genes encoding proteins involved in the transcription and RNA processing of mitochondrial genes (Law et al. 2012). This transient expression was closely followed by an increase in the transcripts encoding proteins involved in the replication and translation of mitochondrial DNA (Law et al. 2012). The expression of genes encoding mitochondrial DNA and RNA metabolism proteins was accompanied by an increase in the components of cytosolic nucleotide biosynthesis and an increase in the members of mitochondrial nucleotide transporter proteins (Law et al. 2012). Only after these transient peaks of expression were typical mitochondrial numbers, morphology and subsequent increase in bioenergetic functions observed, indicating a role for these events in priming translation initiation at the early stages of mitochondrial biogenesis. This was further confirmed by the analysis of protein abundances (using Western blotting and mass spectrometry), which indicated that many proteins display significant positive correlation with transcript abundance during these time points in germination (Law et al. 2012). For example, many components of the electron transport chain and TCA cycle were found to peak in both transcript and protein abundances after $48 \mathrm{~h}$ imbibition (Law et al. 2012).

\section{Biogenesis: transcriptional and post-transcriptional control}

It is readily apparent that mitochondria from different tissues differ in protein composition and abundance, for example, the abundance of glycine decarboxylase in mitochondria from leaves is well documented. Proteomic characterization of mitochondria isolated from different tissues revealed that the abundance of over 200 protein spots differed between tissues (Lee et al. 2008, 2011, 2012). This raises the question of how mitochondrial protein composition is regulated in a cell-specific manner. The studies outlined above showed that there was good coordination between transcript and protein abundances in many cases, indicating that transcriptional regulation plays a role for many mitochondrial proteins (Fig. 1). Furthermore, when comparing transcript and protein abundances and investigating correlations, detailed time courses that account for differences in mRNA and protein turnover need to be taken into account (Law et al. 2012).

In an in-depth study of germination in Arabidopsis, a detailed time course of transcript and protein abundance was analyzed, and it supports the proposal that transcriptional control is an important point of regulation for protein abundance for many, but notably, not all proteins (Narsai et al. 2011; Law et al. 2012). Specifically, one group of proteins that were observed to display low correlation between protein and transcript abundance were the proteins composing the mitochondrial protein import apparatus. It was observed that the protein abundance for these mitochondrial import components increased in abundance over time, while the transcripts encoding these declined several fold (Law et al. 2012). Studies on germination in rice also revealed a similar relationship, with low correlations seen between the abundances of transcripts and proteins of the mitochondrial protein import apparatus (Howell et al. 2006). Notably, these low correlations in transcript versus protein abundances for components of the mitochondrial import apparatus have also been observed in T-DNA mutants, where the inactivation of a gene encoding one isoform of a mitochondrial import component results in an increase in the protein abundance of another isoform, but not the respective transcript abundance (Lister et al. 2007). Thus, it appears that post-transcriptional mechanisms are an important site of regulation for proteins that comprise the mitochondrial protein import apparatus.

While it is readily apparent that transcriptional regulation is an important determinant of mitochondrial protein abundance, only a few factors that regulate nuclear located genes encoding mitochondrial proteins have been identified to date. For example, a detailed study on the regulation of COX5b- 1 revealed AP2/ERF, ABF4, bZIP and trihelix transcription factors as involved in the transcriptional regulation of COX5b-1 (Comelli et al. 2012). Three B3 domain transcription factors (ABI3, FUS3 and LEAFY COTYLEDON2) have also been shown to regulate the expression of the iron-sulphur subunit of complex II during seed development in Arabidopsis (Roschzttardtz et al. 2009). Analysis of the promoters of nuclear genes encoding mitochondrial proteins reveals an enrichment of elements called site II (Gonzalez et al. 2007), that have been subsequently shown to play a key role in the regulation of a variety of nuclear 
genes encoding mitochondrial proteins. Site II elements are cis-acting regulatory elements (CAREs) with the binding sequence of TGGGC(C/T) in genes that are associated with growth, such as the proliferating cell nuclear antigen and ribosomal protein genes (Martin-Trillo and Cubas 2010). Initial studies indicated that these elements may also be involved in the regulation of $C O X 6 b-2, C O X 5 b-2$ and $C y t c$ expression in Arabidopsis (Comelli and Gonzalez 2009; Mufarrege et al. 2009). More detailed studies revealed that site II elements bind various TCP transcription factors involved in the regulation of a variety of nuclear genes encoding mitochondrial proteins (Giraud et al. 2010). Furthermore, via interaction with a number of central clock components, it was demonstrated that diurnal changes in promoter activity, transcript and protein abundance changes could be explained by the regulation of nuclear genes encoding mitochondrial proteins by TCP transcription factors binding to site II elements (Giraud et al. 2010).

The evolution of the plant TCP transcription factor family predates the emergence of land plants, with TCP transcription factors found in simple multicellular green algae with no meristems and no need for complicated organ development of morphologies (Navaud et al. 2007). Therefore, the TCPs present in pre-land plants had a different function to the developmental roles currently accepted for TCPs in higher plants. Thus, the function of the original ancestors of the TCP family is puzzling if they only play a role in development in higher plants. However, the fact that TCP transcription factors regulate energy metabolism in a diurnal manner may represent the original function of TCP transcription factors in plants. It is not possible to determine whether class I or II factors are more related to the ancestral TCPs, as members belonging to both of these distinct groups have been identified in green algae. It is likely that the TCP family and in particular, the CYC/TB subgroup of TCP transcription factors has since expanded in higher plants and evolved to encompass the multitude of roles these factors have in the control of hormone synthesis, morphology and growth (all processes that are linked to the individual environment and the day/night cycles of the circadian clock at some level). The CIN clade of class II factors may be closer to the ancestral group as in Selaginella and Physcomitrella, neither of which have flowers or branches; all class II members are the CIN type (Navaud et al. 2007). TCP2, 3, 11 and 15, which interact with components of the circadian clock (Giraud et al. 2010), all belong to the most ancient classes of TCP factors, the CIN class or PCF class, again reinforcing the hypothesis that these factors originally functioned to control mitochondrial metabolism during cycling environmental conditions. Conservation of the diurnal cycling of transcript profiles for TCPs is conserved across public microarray datasets from Arabidopsis, Populus and rice, with peaks in expression seen at all times across the diurnal cycle, strengthening the case that cycling TCP expression is conserved in higher plants. Also, analysis of the expression of orthologous genes encoding PRR proteins across the same three species revealed a conservation of diurnal rhythms of expression, with sequential peaks seen across the day/night cycle, indicating that the expression pattern of these proposed interacting partners is conserved (Giraud and Whelan, unpublished data). Finally, the finding that a pentatricopeptide repeat protein that is dual targeted to mitochondria and the nucleus (PNM1), and interacts with TCP8 in the nucleus (Hammani et al. 2011a), suggests that the regulation of nuclear genes expression by TCP transcription factors may be coordinated with expression of mitochondrial located genes via pentatricopeptide repeat proteins (Hammani et al. 2011b).

The other extensively studied area examining the regulation of nuclear-encoded mitochondrial gene expression is the induction of mitochondrial proteins and transcripts in response to stress. Analysis of protein and transcript changes in response to individual stresses reveal that under any individual stress, there can be from tens to hundreds of changes (Clifton et al. 2006; Van Aken et al. 2009; Schwarzlander et al. 2012; Tan et al. 2012), but common changes in a number of stresses is relatively small. Apart from the components of the alternative respiratory chain (Clifton et al. 2005), various components associated with protein import also appear to change under stress (Lister et al. 2004). Notably, changes in the latter are often not accompanied by changes in transcript abundance, as outlined above. Overall, the changes in transcript abundance under stress, as determined by array analysis, have been confirmed by quantitative RT-PCR (Clifton et al. 2005, 2006), and/or have been carried out under standard conditions or platforms such as the array Express datasets (Kilian et al. 2007). In contrast, the proteomic studies of the mitochondrial proteome under stress, yields fairly limited insights into protein abundance changes as a result of incomplete coverage (i.e. sensitivity), limited quantification in protein abundance, and lack of confirmation of changes by independent approaches and studies. It is likely that in future, with expansion in the knowledge of the mitochondrial proteome, more proteins and hence genes/transcripts will be added to the list of stress-induced components. However, examples of both the alternative respiratory pathway and components of the protein import apparatus have revealed that transcriptional and post-transcriptional processes operate under stress to drive mitochondrial biogenesis (see below).

The regulation of the alternative respiratory pathway remains one of the best-studied systems for understanding the regulation of nuclear genes encoding mitochondrial proteins. The alternative respiratory pathway consists of a 
terminal cyanide-insensitive terminal oxidase, called the alternative oxidase, and a number of internal and/or external NAD(P)H dehydrogenases (Millar et al. 2011). A variety of treatments have been shown to induce both the transcript and encoded protein abundance for the alternative oxidase (Millar et al. 2011). However, under most of these conditions, an external $\mathrm{NAD}(\mathrm{P}) \mathrm{H}$ dehydrogenase is also induced, and in Arabidopsis, it is NDB2 and AOXIa that are induced to form this alternative respiratory pathway (Clifton et al. 2005). The regulation of this alternative pathway appears to be under quite complex control, apart from transcriptional regulation, post-transcriptional regulatory mechanisms are also involved, as evidenced in a number of studies using salicylic acid (Rhoads and McIntosh 1992; Ho et al. 2008), and also in a dgs 1-1 mutant (Moellering and Benning 2010). AtNCA2 (At5g12290) is an outer mitochondrial membrane protein in Arabidopsis (Duncan et al. 2011), that has also been named DGS-1 (digalactosyl diacylglycerol deficient suppressor 1) in Arabidopsis due to the suppression of the DGD mutant phenotype, through a point mutation near the second transmembrane domain in this gene (referred to as dgs1-1) (Moellering and Benning 2010). However, subsequent studies with an additional null allele have revealed that this effect is independent of the DGS1 protein and that the $d g s 1-1$ phenotype appears to arise from increased levels of hydrogen peroxide, resulting in post-transcriptional repression of the alternative oxidase (Moellering and Benning 2010). DGS1 contains the conserved NCA2 (Nuclear Control of ATPase 2) domain from yeast, which is involved in the regulation of mitochondrial-encoded subunits of the respiratory chain (Camougrand et al. 1995; Pelissier et al. 1995). Interestingly, AtNCA2 (At5g12290) transcript abundance is tightly correlated with genes encoding for proteins involved in signalosome and proteasome regulation (unpublished data). While the mechanism of this signaling/regulatory circuit remains uncharacterised, it indicates a complex system of regulation and cross-talk between the mitochondrion and the nucleus.

Analysis of the direct regulators of the alternative respiratory pathway is still at the early stages, elucidating the factors that signal and regulate expression. While a genetic system to elucidate these factors has been described (Zarkovic et al. 2005), no factors using this system have been described as yet. Other approaches have identified the transcription factor ABI4 as a regulator of alternative oxidase (Giraud et al. 2009), where ABI4 acts as a repressor of alternative oxidase transcription, thus, upon stress treatment the alternative oxidase expression is repressed. The identification of ABI4 as a regulator links mitochondrial and plastid retrograde signaling, as ABI4 also acts as a regulator of plastid retrograde signaling (Koussevitzky et al. 2007).
While the alternative respiratory pathway remains the best-studied system for elucidating factors that regulate the stress induction of mitochondrial transcripts or proteins, there are many other transcripts encoding previously uncharacterised proteins that are also induced upon stress treatment. Initial studies indicate that at least some of these transcripts are induced by different pathways to that of the alternative respiratory components (Ho et al. 2008). Finally, the regulation of genes encoded in the mitochondrion is also an intensively studied aspect of mitochondrial biogenesis. Post-transcriptional modes of regulation are believed to dominate in the regulation of genes located in the mitochondrial genome. Studies of these aspects during mitochondrial biogenesis have been extensively reviewed (Binder and Brennicke 2003; Schmitz-Linneweber and Small 2008).

\section{Mitochondrial biogenesis and activity}

The fact that most mitochondrial proteins must be imported into the mitochondria from the cytosol means that mitochondrial protein import is a vital part of mitochondrial biogenesis. Whilst much of previous work in this area has been focused on identifying the components of the plant mitochondrial import apparatus, and comparing these to their mammalian and fungal counterparts (Carrie et al. 2010; Duncan etal. 2012). The newly identified interaction between the complexes involved in protein import and respiratory chain complexes will be discussed.

Recent analysis of three independent mutants lacking complex I; rug-a nad2 splicing mutant (Kuhn et al. 2011), rpotmp-deficient in a dual-targeted RNA polymerase (Kuhn et al. 2009) and ndufs4 - a structural component of complex I (Kuhn et al. 2009), has shown increased mitochondrial protein import ability and increased abundance of mitochondrial protein import components in all three mutants (Wang et al. 2012). More specifically, all three mutants had a significant increase in the abundance of the inner membrane protein import component Tim23-2 (Wang et al. 2012). Both Tim 23 and Tim17, form the main import channel though the inner mitochondrial membrane, utilized by the majority of proteins destined for the matrix. Further analysis found that an over-expressing (OE) line that increased the amount of Tim23-2 protein by just twofold (Tim23-2 OE), not only exhibited an increase in mitochondrial protein import ability, but also resulted in a reduction in the amount of respiratory complex I (Wang et al. 2012). Furthermore, it was interesting to find that Tim23-2 is not only a part of the Tim17:23 complex, but is also found in the monomeric form of complex I (Wang et al. 2012). In addition, another protein that had previously been defined as a subunit of complex I, named B14.7, and belonging to 
the same family of PRAT (Preprotein and Amino acid Transport family) proteins as Tim23, was found to also associate with Tim17:23 (Wang et al. 2012). Closer analysis of the Tim23-2 OE and rug lines concluded that mitochondrial biogenesis was up-regulated in both lines, as both lines displayed an increase in the transcript abundance for both nuclear and organellar encoded components of the electron transport chain (Wang et al. 2012). This was accompanied by an up-regulation of genes encoding the mitochondrial transcription/translation machinery, mitochondrial import components, and the reactive oxygen species/REDOX scavenging systems in mitochondria. The up-regulation of transcripts encoding components of the mitochondrial transcription/translation machinery was also accompanied by an up-regulation in translation of mitochondrial proteins (Wang et al. 2012) (a phenomenon also witnessed with the rpotmp mutant (Kuhn et al. 2009). Together these data suggest that a disruption of complex I either through direct knockouts or by the over-expression of Tim23-2 results in a signal that induces mitochondrial biogenesis, evidenced by the up-regulation of mitochondrial protein import machinery, induction of transcripts encoding mitochondrial transcription/translation machinery, induction of mitochondrial protein translation, and an induction of electron transport chain components (mitochondrial and nuclear) (Wang et al. 2012).

A direct molecular link/interaction between Tim23-2 and complex I, provides a system by which mitochondrial biogenesis can be regulated. Signals that increase the abundance of Tim23-2 in response to changes in the relative abundance of complex I, either from oxidative damage or increased energy demands, can induce a mitochondrial biogenesis response. However, complex I is not the only respiratory complex that interacts with the Tim17:23 complex (Wang et al. 2012). It has also been observed that Tim23-2 can interact with complex III, and this interaction is mediated through the protein Tim 21 (Wang et al. 2012). In plants, complex III contains the two subunits of the mitochondrial processing peptidase (MPP) (Glaser and Dessi 1999). MPP acts to cleave the mitochondrial targeting peptide from precursor proteins that enter the matrix through the Tim17:23 complex. The role of Tim21 is highlighted by the observations that mutations in Tim21 cause a seedling lethal phenotype (Hamasaki et al. 2012). The Tim21 mutant (called $s d 3$ ) was also found to have lower intracellular ATP levels and result in a dwarf phenotype (Hamasaki et al. 2012). However, when Tim 21 was over-expressed, it caused an increase in cotyledon size in the light, increased hypocotyl thickness in the dark, induction of several transcripts encoding subunits of respiratory complexes II and IV and an increase in intracellular ATP levels (Hamasaki et al. 2012). Thus, several independent studies have linked mitochondrial biogenesis and respiration in mitochondria, which suggests that a retrograde regulatory pathway links mitochondrial biogenesis and activity.

In addition, this type of regulation may also exist in the chloroplast. Over-expression of the chloroplast protein import component Tic40 in Arabidopsis resulted in an upregulation of several other chloroplast inner envelope membrane proteins (Singh et al. 2008). This suggests that the genes encoding these proteins are induced by the overexpression of a chloroplast translocator subunit, likely via an unknown retrograde signal. Based on these studies, it can be proposed that in plants, a retrograde pathway exists from chloroplasts and mitochondria that involve the protein complexes required to import proteins. It is not known exactly how the interactions of these complexes with other components of mitochondria or plastids result in retrograde signaling, but uncovering the existence of such pathway is the first step to the identification of the components involved.

\section{Dual targeting of proteins to mitochondria and plastids/ peroxisomes}

In the last decade it has become apparent that the exclusive targeting of proteins to only one organelle is not a strict rule (Carrie et al. 2009; Carrie and Small 2012). A variety of proteins have now been characterized to be targeted to mitochondria and plastids/peroxisomes, called dual-targeted proteins (Fig. 1) (Carrie et al. 2009; Carrie and Small 2012). With regard to the biological significance of dual targeting and its link to mitochondrial biogenesis, many of these dual-targeted proteins are involved in mitochondrial (and plastid) DNA and RNA metabolism (including replication, transcription and translation). This is of particular interest when investigating mitochondrial biogenesis during seed germination. Recently, it was observed that during germination the growth and division of plastids and mitochondria are taking place concurrently (Law et al. 2012). Specifically, it was seen that early in germination, both mitochondria and plastids are small and less in number, and towards the end of germination they are both more numerous and bigger, taking on their characteristic morphologies (Law et al. 2012). Thus, one role for dual-targeted proteins is that this may coordinate organelle biogenesis during plant development.

The fission of both mitochondria and peroxisomes require dynamins and dynamin-related proteins (DRPs), which are mechano-chemical enzymes or signaling GTPases that form oligomeric rings around membranes (Praefcke and McMahon 2004). At least two DRPs in Arabidopsis, DRP3A and DRP3B, are dual targeted to both mitochondria and peroxisomes (Mano et al. 2004; Fujimoto 
et al. 2009). Loss-of-function mutants of both DRP3A and DRP3B affected the morphologies of both mitochondria and peroxisomes (Mano et al. 2004). While most eukaryotic DRPs lack a transmembrane domain, they are recruited to the membrane by membrane-bound receptors (Praefcke and McMahon 2004). In Arabidopsis, this is believed to be facilitated by FIS1A (BIGYIN) and FIS1B, which are also dual targeted to peroxisomes and mitochondria, and play rate-limiting roles in initiating organelle fission (Scott et al. 2006; Lingard et al. 2008; Zhang and Hu 2008, 2009). The newest member of the peroxisome and mitochondrial division machinery is the peroxisomal and mitochondrial division factor 1 (PMD1), which is dual targeted to the membranes of both mitochondria and peroxisomes. PMD1 is a plant-specific division factor that acts independent of the FIS1-DRP pathway (Aung and $\mathrm{Hu}$ 2011). Plants which lack PMD1 contain enlarged peroxisomes and elongated mitochondria, while over-expression of PMD1 leads to increased numbers of organelles, which are often aggregated (Aung and $\mathrm{Hu}$ 2011). It is interesting to note that PMD1 has a close homolog PMD2, which is only targeted to mitochondria ( $\mathrm{Hu}$ et al. 2012). However, the direct mechanism by which PMD1 and PMD2 impact on mitochondrial and peroxisome division and morphology is still unclear. Notably, the sharing of fission components between mitochondria and peroxisomes is not limited to plants and can be found in animals and fungi as well (Fagarasanu et al. 2007; Kaur and Hu 2009), suggesting these functions are derived from ancient interactions between these organelles.

One of the outstanding questions remaining about dual targeting is: how is it regulated (Fig. 1)? In yeast, it was discovered that the localization of a dual-targeted protein is governed by intracellular metabolite cues (Regev-Rudzki et al. 2009). It has been speculated that this level of control could occur at the organelle surface by having more specific organelle import receptors that allow greater import into that organelle (Carrie and Small 2012). However, as outlined above, the rate of protein import into mitochondria seems to be determined by the inner membrane translocases. Other possible regulatory mechanisms include the phosphorylation of targeting peptides (Lamberti et al. 2011), mRNA targeting to the surface of organelles (Michaud et al. 2010), cellspecific expression of particular import receptors ( $\mathrm{Li}$ and Chiu 2010) or redox regulation of a specific organelles import machineries (Stengel et al. 2010). Additionally, with respect to dual targeting of proteins, it would be beneficial to obtain a more global view of dual-targeted proteins in plants in terms of determining when it arose in plant evolution, and if it is conserved between various species.

\section{Outstanding questions}

In the last decade, progress on the understanding and identification of factors involved in mitochondrial biogenesis have provided greater insights into how mitochondrial function is regulated in a cellular context and given a glimpse of some of the factors involved in this process. It has become clear that mitochondria are not merely passive player(s) that respond to changes in the cellular environment, but rather that they can trigger these changes (Giraud et al. 2008; Van Aken et al. 2009; Vanlerberghe et al. 2009). While the concept of mitochondrial-to-nuclear signaling has been studied from the point of view of regulation of mitochondrial components, the identity of the molecular components that sense mitochondrial function and relay these signals to the nucleus remains unknown. It has been proposed that peptide signaling can provide the specificity in the signaling process (Moller and Sweetlove 2010), and peptide signaling is a well-defined pathway in plant development (Katsir et al. 2011). Such signals would require a specific receptor to give the proposed required specificity. In addition, there are a variety of other signaling pathways that may also exist, and the characterization of the mitochondrial outer membrane proteome from Arabidopsis, and identification of several components potentially associated with signaling (Duncan et al. 2012), provides a platform to increase our understanding of the signaling pathways that exist between mitochondria and other components of the cell. Finding that the protein Sal1, involved in plastid retrograde signaling (Estavillo et al. 2011), is also in present in mitochondria (Estavillo et al. 2011) further emphasizes mitochondrial signaling as an important node of cellular signaling pathways that needs to be further identified and understood, and indicates that it is not a direct linear pathway between mitochondria and the nucleus.

While the identification of individual transcription factors gives insight into how nuclear genes encoding mitochondrial proteins are regulated, as yet it is unknown how mitochondrial biogenesis is triggered. The discovery that there are super complexes or associations between respiratory chain and protein import complexes (Klodmann et al. 2011), and that respiratory chain complexes and protein import complexes share subunits (Wang et al. 2012), also prompts immediate lines of enquiry, which at least link the machines that make mitochondria to mitochondrial activity. The question is: does the dynamic association of these complexes act as a signaling mechanism to trigger mitochondrial biogenesis? During germination, the activation of mitochondrial gene expression appears to occur very early (Law et al. 2012). This raises the question, what role does organellar gene expression play in signaling or regulating the expression of nuclear genes for proteins that may be 
found in the same protein complexes? Further investigations need to be carried out to answer these questions.

Furthermore, a greater understanding of the components of mitochondria is also required, and it is necessary to examine how these components change under a variety of conditions in order to understand the regulation of mitochondrial biogenesis. Thus, unless we have a complete "parts" list of mitochondria, it will be difficult to understand how it is assembled and how it changes. This "parts list" is also important to gain understanding into the relationships with other organelles. While it was once believed that proteins have one sub-cellular location in a cell, the realization that dual targeting may be quite common raises another level of complexity and regulation. It is unlikely that the partitioning of dual-targeted proteins is not regulated; however, as yet, we have no insight into the mechanism(s) by which this may occur.

Thus, there is still some way to go to in order to understand how plants make mitochondria. While Arabidopsis has provided a valuable model in the last decade, the advent of next generation sequencing, means that a variety of other plants that may be more appropriate for more specific aspects may now be utilized to gain insights into how plants make mitochondria.

Acknowledgments C.C. is supported by a Humboldt Research Fellowship for Postdoctoral Researchers from the Alexander von Humboldt Foundation. J.W. is supported by an Australian Research Council Centre of Excellence Program CEO561495. M.W.M. is supported by an Australian Research Council Australian Postdoctoral Fellowship grant.

\section{References}

Aung K, Hu J (2011) The Arabidopsis tail-anchored protein PEROXISOMAL AND MITOCHONDRIAL DIVISION FACTOR1 is involved in the morphogenesis and proliferation of peroxisomes and mitochondria. Plant Cell 23:4446-4461

Binder S, Brennicke A (2003) Gene expression in plant mitochondria: transcriptional and post-transcriptional control. Philos Trans R Soc Lond B Biol Sci 358:181-188 discussion 188-189

Camougrand N, Pelissier P, Velours G, Guerin M (1995) NCA2, a second nuclear gene required for the control of mitochondrial synthesis of subunits 6 and 8 of ATP synthase in Saccharomyces cerevisiae. J Mol Biol 247:588-596

Carrie C, Small I (2012) A reevaluation of dual-targeting of proteins to mitochondria and chloroplasts. Biochim Biophys Acta (PMID:22683762)

Carrie C, Giraud E, Whelan J (2009) Protein transport in organelles: dual targeting of proteins to mitochondria and chloroplasts. FEBS J 276:1187-1195

Carrie C, Murcha MW, Whelan J (2010) An in silico analysis of the mitochondrial protein import apparatus of plants. BMC Plant Biol 10:249

Clifton R, Lister R, Parker KL, Sappl PG, Elhafez D, Millar AH, Day DA, Whelan J (2005) Stress-induced co-expression of alternative respiratory chain components in Arabidopsis thaliana. Plant Mol Biol 58:193-212
Clifton R, Millar AH, Whelan J (2006) Alternative oxidases in Arabidopsis: a comparative analysis of differential expression in the gene family provides new insights into function of non-phosphorylating bypasses. Biochim Biophys Acta 1757:730-741

Comelli RN, Gonzalez DH (2009) Identification of regulatory elements involved in expression and induction by sucrose and UVB light of the Arabidopsis thaliana COX5b-2 gene, encoding an isoform of cytochrome $\mathrm{c}$ oxidase subunit $5 \mathrm{~b}$. Physiol Plant 137:213-224

Comelli RN, Welchen E, Kim HJ, Hong JC, Gonzalez DH (2012) Delta subclass HD-Zip proteins and a B-3 AP2/ERF transcription factor interact with promoter elements required for expression of the Arabidopsis cytochrome c oxidase 5b-1 gene. Plant Mol Biol 80:157-167

Duncan O, Taylor NL, Carrie C, Eubel H, Kubiszewski-Jakubiak S, Zhang B, Narsai R, Millar AH, Whelan J (2011) Multiple lines of evidence localize signaling, morphology, and lipid biosynthesis machinery to the mitochondrial outer membrane of Arabidopsis. Plant Physiol 157:1093-1113

Duncan O, Murcha MW, Whelan J (2012) Unique components of the plant mitochondrial protein import apparatus. Biochim Biophys Acta (PMID:22406071)

Ehrenshaft M, Brambl R (1990) Respiration and mitochondrial biogenesis in germinating embryos of maize. Plant Physiol 93:295-304

Estavillo GM, Crisp PA, Pornsiriwong W, Wirtz M, Collinge D, Carrie C, Giraud E, Whelan J, David P, Javot H, Brearley C, Hell R, Marin E, Pogson BJ (2011) Evidence for a SAL1-PAP chloroplast retrograde pathway that functions in drought and high light signaling in Arabidopsis. Plant Cell 23:3992-4012

Fagarasanu A, Fagarasanu M, Rachubinski RA (2007) Maintaining peroxisome populations: a story of division and inheritance. Annu Rev Cell Dev Biol 23:321-344

Fujimoto M, Arimura S, Mano S, Kondo M, Saito C, Ueda T, Nakazono M, Nakano A, Nishimura M, Tsutsumi N (2009) Arabidopsis dynamin-related proteins DRP3A and DRP3B are functionally redundant in mitochondrial fission, but have distinct roles in peroxisomal fission. Plant J 58:388-400

Giraud E, Ho LH, Clifton R, Carroll A, Estavillo G, Tan YF, Howell KA, Ivanova A, Pogson BJ, Millar AH, Whelan J (2008) The absence of ALTERNATIVE OXIDASE1a in Arabidopsis results in acute sensitivity to combined light and drought stress. Plant Physiol 147:595-610

Giraud E, Van Aken O, Ho LH, Whelan J (2009) The transcription factor ABI4 is a regulator of mitochondrial retrograde expression of ALTERNATIVE OXIDASE1a. Plant Physiol 150:1286-1296

Giraud E, Ng S, Carrie C, Duncan O, Low J, Lee CP, Van Aken O, Millar AH, Murcha M, Whelan J (2010) TCP transcription factors link the regulation of genes encoding mitochondrial proteins with the circadian clock in Arabidopsis thaliana. Plant Cell 22:3921-3934

Glaser E, Dessi P (1999) Integration of the mitochondrial-processing peptidase into the cytochrome bc1 complex in plants. J Bioenerg Biomembr 31:259-274

Gonzalez DH, Welchen E, Attallah CV, Comelli RN, Mufarrege EF (2007) Transcriptional coordination of the biogenesis of the oxidative phosphorylation machinery in plants. Plant J 51:105-116

Hamasaki H, Yoshizumi T, Takahashi N, Higuchi M, Kuromori T, Imura Y, Shimada H, Matsui M (2012) SD3, an Arabidopsis thaliana homolog of TIM21, affects intracellular ATP levels and seedling development. Mol Plant 5:461-471

Hammani K, Gobert A, Hleibieh K, Choulier L, Small I, Giegé P (2011a) An Arabidopsis dual-localized pentatricopeptide repeat protein interacts with nuclear proteins involved in gene expression regulation. Plant Cell 23:730-740

Hammani K, Gobert A, Small I, Giegé P (2011b) A PPR protein involved in regulating nuclear genes encoding mitochondrial proteins? Plant Signal Behav 6:748-750 
Ho LH, Giraud E, Uggalla V, Lister R, Clifton R, Glen A, ThirkettleWatts D, Van Aken O, Whelan J (2008) Identification of regulatory pathways controlling gene expression of stress-responsive mitochondrial proteins in Arabidopsis. Plant Physiol 147: 1858-1873

Howell KA, Millar AH, Whelan J (2006) Ordered assembly of mitochondria during rice germination begins with pro-mitochondrial structures rich in components of the protein import apparatus. Plant Mol Biol 60:201-223

Howell KA, Millar AH, Whelan J (2007) Building the powerhouse: what are the signals involved in plant mitochondrial biogenesis? Plant Signal Behav 2:428-430

Hu J, Baker A, Bartel B, Linka N, Mullen RT, Reumann S, Zolman BK (2012) Plant peroxisomes: biogenesis and function. Plant Cell 24:2279-2303

Katsir L, Davies KA, Bergmann DC, Laux T (2011) Peptide signaling in plant development. Curr Biol 21:R356-R364

Kaur N, Hu J (2009) Dynamics of peroxisome abundance: a tale of division and proliferation. Curr Opin Plant Biol 12:781-788

Kilian J, Whitehead D, Horak J, Wanke D, Weinl S, Batistic O, D'Angelo C, Bornberg-Bauer E, Kudla J, Harter K (2007) The AtGenExpress global stress expression data set: protocols, evaluation and model data analysis of UV-B light, drought and cold stress responses. Plant J 50:347-363

Klodmann J, Senkler M, Rode C, Braun HP (2011) Defining the protein complex proteome of plant mitochondria. Plant Physiol 157:587-598

Koussevitzky S, Nott A, Mockler TC, Hong F, Sachetto-Martins G, Surpin M, Lim J, Mittler R, Chory J (2007) Signals from chloroplasts converge to regulate nuclear gene expression. Science 316:715-719

Kuhn K, Richter U, Meyer EH, Delannoy E, de Longevialle AF, O'Toole N, Borner T, Millar AH, Small ID, Whelan J (2009) Phagetype RNA polymerase RPOTmp performs gene-specific transcription in mitochondria of Arabidopsis thaliana. Plant Cell 21:2762-2779

Kuhn K, Carrie C, Giraud E, Wang Y, Meyer EH, Narsai R, des Francs-Small CC, Zhang B, Murcha MW, Whelan J (2011) The $\mathrm{RCC} 1$ family protein RUG3 is required for splicing of $\mathrm{nad} 2$ and complex I biogenesis in mitochondria of Arabidopsis thaliana. Plant J 67:1067-1080

Lamberti G, Drurey C, Soll J, Schwenkert S (2011) The phosphorylation state of chloroplast transit peptides regulates preprotein import. Plant Signal Behav 6:1918-1920

Law SR, Narsai R, Taylor NL, Delannoy E, Carrie C, Giraud E, Millar AH, Small I, Whelan J (2012) Nucleotide and RNA metabolism prime translational initiation in the earliest events of mitochondrial biogenesis during Arabidopsis germination. Plant Physiol 158:1610-1627

Lee CP, Eubel H, O'Toole N, Millar AH (2008) Heterogeneity of the mitochondrial proteome for photosynthetic and non-photosynthetic Arabidopsis metabolism. Mol Cell Proteomics 7:12971316

Lee CP, Eubel H, O'Toole N, Millar AH (2011) Combining proteomics of root and shoot mitochondria and transcript analysis to define constitutive and variable components in plant mitochondria. Phytochemistry 72:1092-1108

Lee CP, Eubel H, Solheim C, Millar AH (2012) Mitochondrial proteome heterogeneity between tissues from the vegetative and reproductive stages of Arabidopsis thaliana development. J Proteome Res (PMID:22540835)

Li HM, Chiu CC (2010) Protein transport into chloroplasts. Annu Rev Plant Biol 61:157-180

Lingard MJ, Gidda SK, Bingham S, Rothstein SJ, Mullen RT, Trelease RN (2008) Arabidopsis PEROXIN11c-e, FISSION1b, and DYN-
AMIN-RELATED PROTEIN3A cooperate in cell cycle-associated replication of peroxisomes. Plant Cell 20:1567-1585

Lister R, Chew O, Lee MN, Heazlewood JL, Clifton R, Parker KL, Millar AH, Whelan J (2004) A transcriptomic and proteomic characterization of the Arabidopsis mitochondrial protein import apparatus and its response to mitochondrial dysfunction. Plant Physiol 134:777-789

Lister R, Carrie C, Duncan O, Ho LH, Howell KA, Murcha MW, Whelan J (2007) Functional definition of outer membrane proteins in volved in preprotein import into mitochondria. Plant Cell 19:3739-3759

Logan DC (2010) The dynamic plant chondriome. Semin Cell Dev Biol 21:550-557

Logan DC, Millar AH, Sweetlove LJ, Hill SA, Leaver CJ (2001) Mitochondrial biogenesis during germination in maize embryos. Plant Physiol 125:662-672

Mano S, Nakamori C, Kondo M, Hayashi M, Nishimura M (2004) An Arabidopsis dynamin-related protein, DRP3A, controls both peroxisomal and mitochondrial division. Plant J 38:487-498

Martin-Trillo M, Cubas P (2010) TCP genes: a family snapshot ten years later. Trends Plant Sci 15:31-39

Michaud M, Marechal-Drouard L, Duchene AM (2010) RNA trafficking in plant cells: targeting of cytosolic mRNAs to the mitochondrial surface. Plant Mol Biol 73:697-704

Millar AH, Whelan J, Soole KL, Day DA (2011) Organization and regulation of mitochondrial respiration in plants. Annu Rev Plant Biol 62:79-104

Moellering ER, Benning C (2010) Phosphate regulation of lipid biosynthesis in Arabidopsis is independent of the mitochondrial outer membrane DGS1 complex. Plant Physiol 152:1951-1959

Moller IM, Sweetlove LJ (2010) ROS signalling —-specificity is required. Trends Plant Sci 15:370-374

Mufarrege EF, Curi GC, Gonzalez DH (2009) Common sets of promoter elements determine the expression characteristics of three Arabidopsis genes encoding isoforms of mitochondrial cytochrome c oxidase subunit 6b. Plant Cell Physiol 50:1393-1399

Narsai R, Law SR, Carrie C, Xu L, Whelan J (2011) In-depth temporal transcriptome profiling reveals a crucial developmental switch with roles for RNA processing and organelle metabolism that are essential for germination in Arabidopsis. Plant Physiol 157:13421362

Navaud O, Dabos P, Carnus E, Tremousaygue D, Herve C (2007) TCP transcription factors predate the emergence of land plants. J Mol Evol 65:23-33

Nisoli E, Clementi E, Moncada S, Carruba MO (2004) Mitochondrial biogenesis as a cellular signaling framework. Biochem Pharmacol 67:1-15

Pelissier P, Camougrand N, Velours G, Guerin M (1995) NCA3, a nuclear gene involved in the mitochondrial expression of subunits 6 and 8 of the Fo-F1 ATP synthase of $S$. cerevisiae. Curr Genet 27:409-416

Peters K, Niessen M, Peterhansel C, Spath B, Holzle A, Binder S, Marchfelder A, Braun HP (2012) Complex I-complex II ratio strongly differs in various organs of Arabidopsis thaliana. Plant Mol Biol 79:273-284

Plattner H, Salpeter MM, Saltzgaber J, Schatz G (1970) Promitochondria of anaerobically grown yeast. IV. Conversion into respiring mitochondria. Proc Natl Acad Sci USA 66:1252-1259

Praefcke GJ, McMahon HT (2004) The dynamin superfamily: universal membrane tubulation and fission molecules? Nat Rev Mol Cell Biol 5:133-147

Prasad TK, Stewart CR (1992) cDNA clones encoding Arabidopsis thaliana and Zea mays mitochondrial chaperonin HSP60 and gene expression during seed germination and heat shock. Plant Mol Biol 18:873-885 
Preuten T, Cincu E, Fuchs J, Zoschke R, Liere K, Borner T (2010) Fewer genes than organelles: extremely low and variable gene copy numbers in mitochondria of somatic plant cells. Plant J 64:948-959

Regev-Rudzki N, Battat E, Goldberg I, Pines O (2009) Dual localization of fumarase is dependent on the integrity of the glyoxylate shunt. Mol Microbiol 72:297-306

Rhoads DM, McIntosh L (1992) Salicylic acid regulation of respiration in higher plants: alternative oxidase expression. Plant Cell 4:1131-1139

Roschzttardtz H, Fuentes I, Vasquez M, Corvalan C, Leon G, Gomez I, Araya A, Holuigue L, Vicente-Carbajosa J, Jordana X (2009) A nuclear gene encoding the iron-sulfur subunit of mitochondrial complex II is regulated by B3 domain transcription factors during seed development in Arabidopsis. Plant Physiol 150:84-95

Schmitz-Linneweber C, Small I (2008) Pentatricopeptide repeat proteins: a socket set for organelle gene expression. Trends Plant Sci 13:663-670

Schwarzlander M, Konig AC, Sweetlove LJ, Finkemeier I (2012) The impact of impaired mitochondrial function on retrograde signalling: a meta-analysis of transcriptomic responses. J Exp Bot 63:1735-1750

Scott I, Tobin AK, Logan DC (2006) BIGYIN, an orthologue of human and yeast FIS1 genes functions in the control of mitochondrial size and number in Arabidopsis thaliana. J Exp Bot 57:1275-1280

Singh ND, Li M, Lee SB, Schnell D, Daniell H (2008) Arabidopsis Tic40 expression in tobacco chloroplasts results in massive proliferation of the inner envelope membrane and upregulation of associated proteins. Plant Cell 20:3405-3417

Stengel A, Benz JP, Soll J, Bolter B (2010) Redox-regulation of protein import into chloroplasts and mitochondria: similarities and differences. Plant Signal Behav 5:105-109
Tan YF, Millar AH, Taylor NL (2012) Components of mitochondrial oxidative phosphorylation vary in abundance following exposure to cold and chemical stresses. J Proteome Res 11:3860-3879

Thompson P, Bowsher CG, Tobin AK (1998) Heterogeneity of mitochondrial protein biogenesis during primary leaf development in barley. Plant Physiol 118:1089-1099

Topping JF, Leaver CJ (1990) Mitochondrial gene expression during wheat leaf development. Planta 182:399-407

Van Aken O, Zhang B, Carrie C, Uggalla V, Paynter E, Giraud E, Whelan J (2009) Defining the mitochondrial stress response in Arabidopsis thaliana. Mol Plant 2:1310-1324

Vanlerberghe GC, Cvetkovska M, Wang J (2009) Is the maintenance of homeostatic mitochondrial signaling during stress a physiological role for alternative oxidase? Physiol Plant 137:392-406

Wang Y, Carrie C, Giraud E, Elhafez D, Narsai R, Duncan O, Whelan J, Murcha MW (2012) Dual location of the mitochondrial preprotein transporters B14.7 and Tim23-2 in complex I and the TIM17:23 complex in Arabidopsis links mitochondrial activity and biogenesis. Plant Cell 24:2675-2695

Zarkovic J, Anderson SL, Rhoads DM (2005) A reporter gene system used to study developmental expression of alternative oxidase and isolate mitochondrial retrograde regulation mutants in Arabidopsis. Plant Mol Biol 57:871-888

Zhang XC, Hu JP (2008) FISSION1A and FISSION1B proteins mediate the fission of peroxisomes and mitochondria in Arabidopsis. Mol Plant 1:1036-1047

Zhang X, Hu J (2009) Two small protein families, DYNAMIN-RELATED PROTEIN3 and FISSION1, are required for peroxisome fission in Arabidopsis. Plant J 57:146-159 